\title{
Inmunoexpresión de p53 y ciclina D1 en adenomas de vesícula biliar
}

\author{
F. Arévalo, C. Arias Stella y E. Monge ${ }^{1}$ \\ Instituto de Anatomía Patológica y Biológica Molecular "Arias Stella”. 'Servicio de Gastroenterología Daniel Alcides \\ Carrión. Universidad Nacional Mayor de San Marcos y Departamento de Fisiología Universidad Cayetano Heredia. \\ Lima, Perú
}

\section{RESUMEN}

Introducción: el adenoma de vesícula biliar es una neoplasia infrecuente, cuya relación con el adenocarcinoma es poco conoci$\mathrm{da}$, aunque algunos autores han propuesto que la mayoría de adenomas no degeneran en adenocarcinomas, debido a que ambas lesiones presentan vías moleculares diferentes.

Material y métodos: el presente trabajo es un estudio transversal que compara las características moleculares del adenoma y adenocarcinoma de vesícula biliar, mediante la medición inmunohistoquímica de la expresión de las proteína p53 y ciclina D1 (ambas reguladoras del ciclo celular) en 12 enfermos de cada grupo.

Resultados: encontramos una mayor expresión de p53 en los adenocarcinomas $(83,3 \%)$ que en los adenomas $(16,6 \%)$ siendo esta diferencia estadísticamente significativa usando el test de chi cuadrado $(p=0,003)$, mientras que la expresión de ciclina D1 en ambos grupos fue similar.

Conclusión: consideramos que nuestros resultados indican que la alteración en el p53 es un paso importante en el desarrollo de los adenocarcinomas de vesícula biliar, mientras que en el desarrollo de los adenomas, la alteración del p53 sería poco trascendente. Por otro lado, la sobreexpresión de ciclina D1 sería un mecanismo molecular común a ambas lesiones.

Palabras clave: Adenoma de vesícula biliar. Adenocarcinoma de vesícula biliar. p53. Ciclina D1.

\begin{abstract}
Introduction: gallbladder adenomas are infrequent neoplasms whose relation to adenocarcinoma is not well understood. It has been suggested that adenomas and adenocarcinomas follow different molecular pathways.

Material and methods: this is a comparative, cross-sectional study in which we compared p53 and D1 cyclin expression in adenomas and adenocarcinomas of the gallbladder.

Results: we included 12 cases in each group. Expression of p53 occurred in $83.3 \%$ of adenocarcinomas and in $16.6 \%$ of adenomas $(p=0.003)$. D1 cyclin was expressed in a similar number of adenomas and adenocarcinomas.

Conclusion: our results support the hypothesis that p53 is an important step in the pathogenesis of adenocarcinomas but not of adenomas of the gallbladder. D1 cyclin is apparently a common pathway involved in the genesis of both tumors.
\end{abstract}

Key words: Gallbladder adenoma. Gallbladder adenocarcinoma. p53. Cyclin D1.

Arévalo F, Arias Stella CJ, Monge E. Inmunoexpresión de p53 y ciclina D1 en adenomas de vesícula biliar. Rev Esp Enferm Dig 2007; 99: 694-697.

\section{INTRODUCCIÓN}

Los adenomas de vesícula biliar son neoplasias infrecuentes que se observan en menos del $1 \%$ de las colecis-

Recibido: 24-09-07.

Aceptado: 01-10-07.

Correspondencia: Fernando Arévalo. Servicio de Anatomía Patológica. Instituto Arias Stella. Av. Gregorio Escobedo 612. Jesús María, Lima. Perú.e-mail: fer13013@hotmail.com tectomías. Por su baja frecuencia, son pocos los trabajos que se han realizado sobre estos adenomas; a pesar de ello, la mayoría de autores consideran que sólo un muy pequeño porcentaje de adenomas de vesícula biliar degeneran en carcinomas (1).

La proteína p53 es un oncosupresor tumoral que se encuentra alterado en aproximadamente la mitad de los casos de cáncer de vesícula, mientras que la ciclina D1, una proteína que controla el ciclo celular, se ha encontrado sobreexpresada con mayor frecuencia en los adenomas que en los adenocarcinomas de vesícula biliar (2). Consi- 
deramos que si los adenomas y adenocarcinomas formaran parte de una misma secuencia oncogénica deberían compartir similares características moleculares.

El objetivo del presente trabajo fue el de evaluar si existen diferencias o semejanzas en la inmunoexpresión de p53 y ciclina D1 en adenomas y en adenocarcinomas.

\section{MATERIALES Y MÉTODOS}

De todas las piezas de colecistectomía enviadas al Instituto Arias Stella durante los años 2005 y 2006 se seleccionaron todos los casos de adenoma (12 casos) y de adenocarcinoma (16 casos); sin embargo, del grupo de los adenocarcinomas solamente se pudieron utilizar las muestras de 12 casos, ya que no se disponía de suficiente material. Se consideró como adenoma de vesícula biliar a toda formación elevada constituida histológicamente por una proliferación glandular con algún grado de displasia; por el contrario, se diagnosticó como adenocarcinoma de vesícula biliar a aquellas formaciones en las que existían células atípicas formando glándulas y cuyo origen del epitelio de superficie vesicular, pueda observarse microscópicamente.

Se midieron las siguientes variables: inmunoexpresión de p53 y de ciclina D1, sexo, edad y tamaño de la lesión. Las láminas y los bloques de parafina se obtuvieron del archivo del instituto.

La presencia de p53 y ciclina D1 se determinó mediante técnicas de inmunoperoxidasa. Para conocer la inmunoexpresión del p53 se emplearon anticuerpos monoclonales de ratón, clona D0-7 (DAKO). La inmunoexpresión de ciclina D1 se reconoció usando anticuerpos monoclonales de conejo, clona RBT-14 (CELLMARCK). Se catalogó como célula positiva para p53 y ciclina D1 a aquella que presentaba coloración dorada nuclear después del procesamiento inmunohistoquímico y como caso positivo para p53 y ciclina D1 a aquel que presentaba más del 15\% de la población celular positiva para $\mathrm{p} 53$. El porcentaje de positividad inmunohistoquímica se determinó contando el número de células positivas inmnohistoquímicamente en 3 campos de 40X, en microscopios Leica y Olympus, realizado por patólogos del Instituto Arias Stella. Los resultados se expresaron en porcentajes y fueron analizados con ayuda del programa Excel y SPSS 11.0.

\section{RESULTADOS}

Entre un total de 1.798 vesículas recibidas durante el año 2005-2006, se encontraron 12 adenomas $(0,66 \%)$ y 16 adenocarcinomas $(0,8 \%)$. La tabla I recoge los porcentajes de estas dos lesiones así como los de otras enfermedades existentes en las vesículas recibidas.

La edad promedio de los pacientes portadores de adenoma fue de 43,2 años y el 91,6\% de ellos eran mujeres. El tamaño promedio de los adenomas fue de $1,008 \mathrm{~cm}$ y sólo 2 casos de adenoma presentaron focos de microcarcinoma.
Tabla I. Patología de vesícula biliar

\begin{tabular}{lcc}
\hline & \multicolumn{2}{c}{$2005-2006$} \\
& $n$ & $\%$ \\
\hline Colesterolosis & 436 & 24,2 \\
Colecistitis aguda & 398 & 22,1 \\
Adenomiosis & 62 & 3,4 \\
Adenocarcinoma & 16 & 0,8 \\
Pólipo adenomatoso & 12 & 0,6 \\
Pólipo hiperplásico & 9 & 0,5 \\
Total & 1.798 & 100 \\
\hline
\end{tabular}

La inmunoexpresión de ciclina D1 fue similar en los adenomas y en los adenocarcinomas (Tabla II y Fig. 2).

Con el fin de comparar la inmunoexpresión de p53 existente en los adenocarcinomas con la presente en los adenomas, se elaboró un cuadro de contingencia. Usando el test de Fisher, se obtuvo una diferencia que fue significativa $(\mathrm{p}=0,003)($ Tabla III y Figs. 1, 3, 4 y 5).

\begin{tabular}{lccccc}
\multicolumn{5}{c}{ Tabla II. Inmunoexpresión de ciclina D1 en adenomas y } \\
adenocarcinomas \\
\hline \multicolumn{5}{c}{ Ciclina D1 } \\
& Positivo (\%) & Negativo (\%) & Total \\
\hline Adenocarcinoma & 7 & 58,3 & 5 & 41,6 & 12 \\
Adenoma & 8 & 66,6 & 4 & 33,3 & 12 \\
\hline
\end{tabular}

Tabla III. Inmunoexpresión del p53 en adenomas y en adenocarcinomas

\begin{tabular}{lcccccc}
\hline & \multicolumn{4}{c}{$p 53$} & & \\
& \multicolumn{1}{c}{ Positivo (\%) } & \multicolumn{2}{c}{ Negativo (\%) } & Total \\
\hline Adenocarcinoma & 10 & 83,3 & 2 & 16,6 & 12 \\
Adenoma & 2 & 16,6 & & 10 & 83,3 & 12 \\
\hline $\mathrm{p}=0,003$. & & & & &
\end{tabular}

\section{DISCUSIÓN}

La ciclina D1 es una proteína que controla el ciclo celular, permitiendo la progresión desde la fase G1 a la fase $\mathrm{S}$ del ciclo celular. Siendo este su efecto, es evidente que una sobreexpresión anormal de la ciclina D1 debe conducir a una proliferación celular. Una sobreexpresión de la ciclina D1 ha sido hallada en neoplasias tales como los linfomas, cáncer de colon y de mama y en algunas lesiones preneoplásicas como, por ejemplo, en pólipos adenomatosos de colon y en la hiperplasia ductal de la mama (2).

Existen pocos trabajos en los que haya estudiado la expresión de la ciclina D1 en adenomas y adenocarcinomas de vesícula biliar. En un trabajo realizado en Japón (2), se describe que la expresión de la ciclina D1 es mayor en los adenomas (67\%) que en los adenocarcinomas (41\%). Por el contrario, en un trabajo procedente de China (3), se menciona que esa expresión es mayor en los adenocarcinomas $(68,3 \%)$ que en los adenomas (57\%). En nuestra 


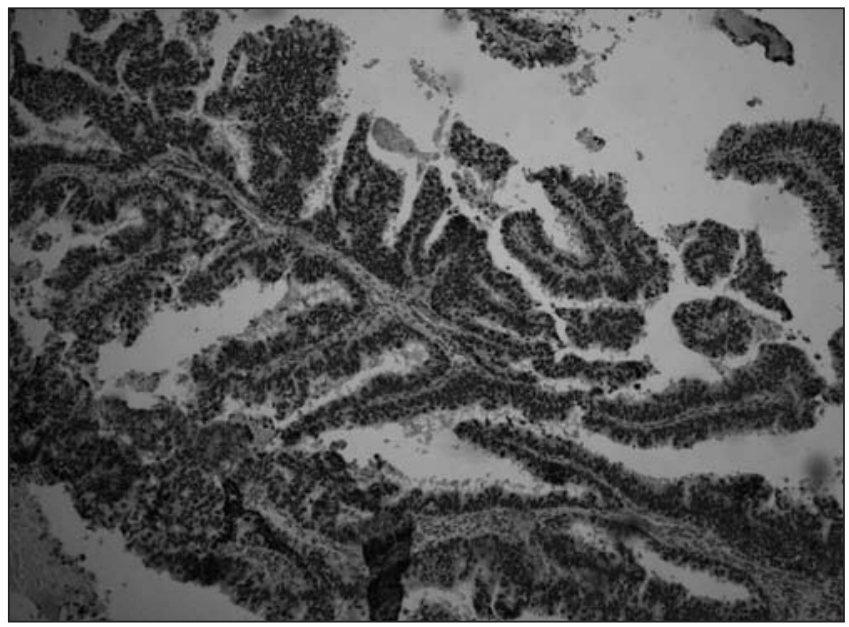

Fig. 1. Adenocarcinoma infiltrante positivo para el p53. Inmunoperoxidasa $10 \mathrm{X}$.

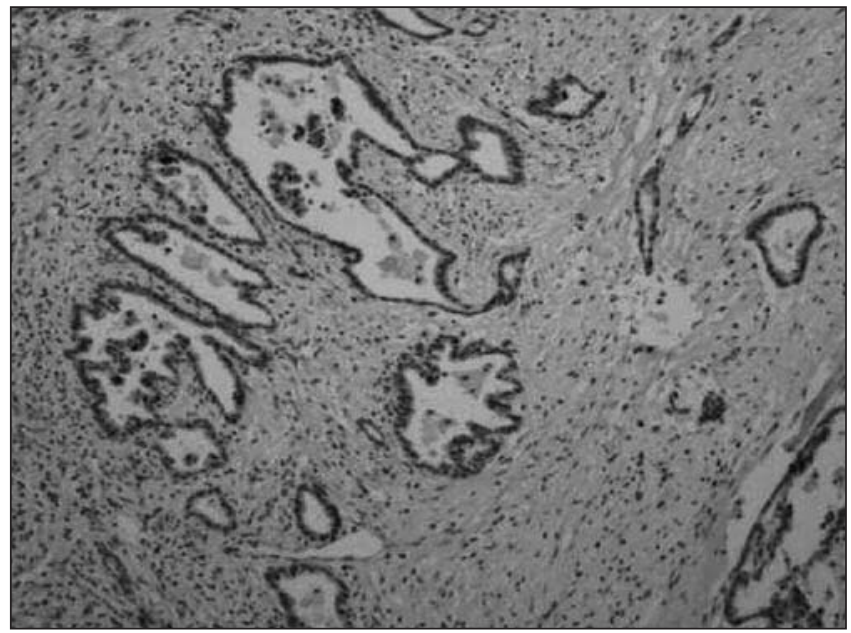

Fig. 3. Adenoma negativo para el p53. Inmunoperoxidasa 40X.

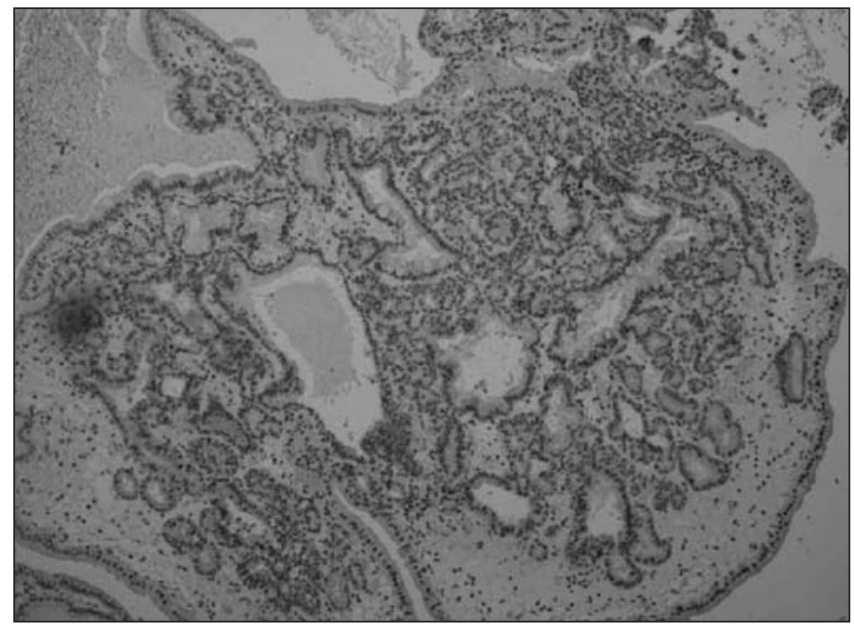

Fig. 2. Adenoma positivo para ciclina D1-4X.

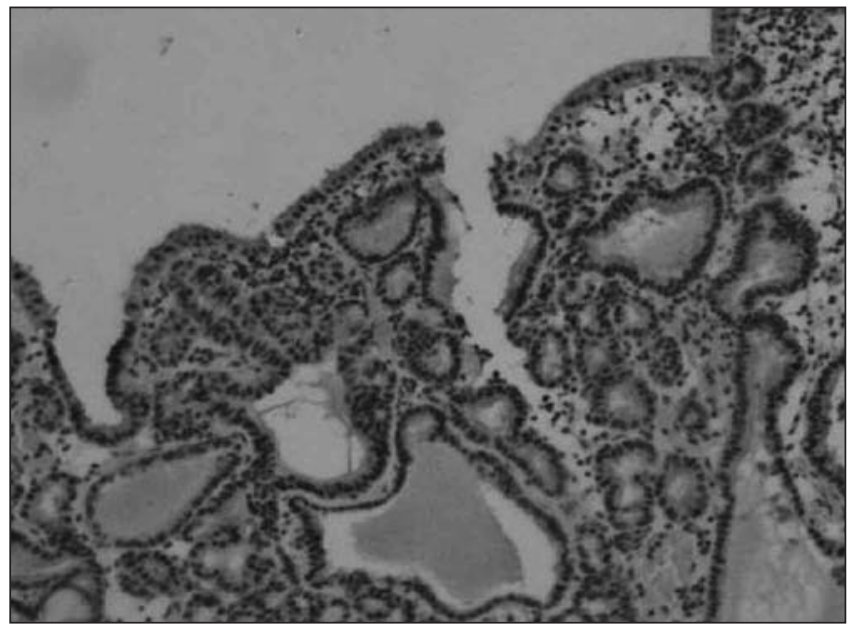

Fig. 4. Adenoma negativo para el p53. 40X.

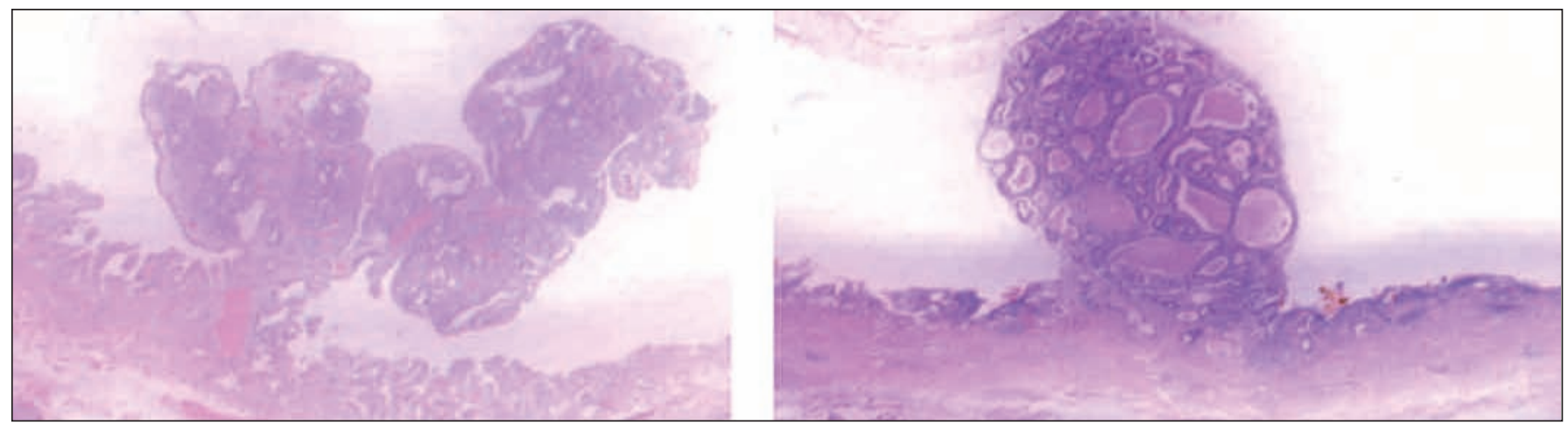

Fig. 5. Vista panorámica de 2 casos de adenoma de vesícula biliar. HE.

experiencia, la sobreexpresión de la ciclina D1 tiene lugar tanto en los adenomas $(66,6 \%)$ como en los adenocarcinomas $(58,3 \%)$ y en ambos esta sobreexpresión ocurre con una frecuencia similar. Nuestros resultados, junto con los de otros autores, nos sugieren que la sobreexpresión de ciclina D1 es una característica molecular común que se encuentra tanto en los adenomas como en los adenocarcinomas. 
En el estudio japonés arriba mencionado se plantea la posibilidad de que la sobreexpresión de ciclina D1 participe en la conversión de adenoma en adenocarcinoma de vesícula. En uno de nuestros casos de adenoma positivo inmunohistoquímicamente para ciclina D1 encontramos focos de microcarcinoma; sin embargo, esta positividad para la ciclina D1 fue también observada en casos de adenomas sin carcinoma, en los que la positividad para ciclina D1 fue incluso más intensa y difusa. Consideramos que la ciclina D1 participa en el desarrollo de los adenomas, pero, al menos en nuestros casos, sería otro el mecanismo molecular responsable de la transformación maligna.

La proteína p53 es un regulador del ciclo celular y una de las dianas que más frecuentemente se alteran en la mayoría de neoplasias del ser humano. Su función consiste en mantener la integridad del ADN y en inducir la apoptosis de las células cuyo ADN sea anormal e imposible de reparar (4). De ahí su importancia en el desarrollo de los carcinomas, ya que un fracaso funcional del p53 permitiría la persistencia de ADN anómalo y la perpetuación de poblaciones celulares anormales, con potencial maligno, debido al fracaso de la apoptosis.

El análisis inmunohistoquímico empleado permite detectar cúmulos de p53, que normalmente no se depositan, por ello se asume que el p53 detectado por la inmunohistoquímica es un p53 alterado. Este método tiene una concordancia del $80 \%$ con el método de secuenciación génica utilizado para detectar mutaciones en el gen p53; por ello se acepta que este método ofrece una fiabilidad aceptable (4).

En el tracto digestivo, son comunes las alteraciones del p53; en especial son particularmente frecuentes en el adenocarcinoma de colon (60 a 70\%), de estómago (20 a $50 \%$ ) y en el carcinoma de esófago (5).

En la vesícula biliar, la inmunoexpresión del p53 ha sido encontrada, por diferentes autores, más frecuentemente en los adenocarcinomas (35 a 65\%) (4) que en los adenomas (0 al 17\%) (6-9). Igualmente, nosotros encontramos que el p53 se encuentra alterado más frecuentemente en los adenocarcinomas $(83,3 \%)$ que en los adenomas $(16,6 \%)$.

Esta diferencia en la inmunoexpresión del p53 entre adenomas y adenocarcinomas, puede tener 2 interpretaciones. En primer lugar, es posible que los adenomas desarrollen mutaciones en el gen del p53 tardíamente, en el proceso de transición a adenocarcinoma. De esta forma estas mutaciones sean difíciles de detectar inicialmente en el momento que se hace el diagnóstico en la pieza quirúrgica. Sin embargo, la ausencia de inmunoexpresión de p53 en casos de adenoma con focos de microcarcinoma contradice tal hipótesis. Por ello, creemos que la transformación cancerígena de los adenomas no seguiría la vía del p53 sino otras vías. Esto nos lleva a la segunda interpretación. En segundo lugar, es posible que, la altera- ción del p53 sea un evento molecular característico de la mayoría de adenocarcinomas de vesícula biliar pero innecesario en el desarrollo de los adenomas, debido posiblemente a que estos dos tumores tengan orígenes moleculares diferentes. Esta idea también ha sido planteada por otros autores; en efecto, Witusba y cols. (10), estudiando la presencia de mutaciones de p53, Kras y Nras y pérdida de heterozigosidad, concluyen que los adenomas de vesícula biliar presentan anormalidades moleculares diferentes que las detectadas en los carcinomas de vesícula biliar.

La ausencia de alteraciones en el p53 en los adenomas de vesícula implicaría que deben de existir otras vías moleculares que permitan la degeneración maligna de estos adenomas. Esta vía molecular alternativa y desconocida tendría aparentemente un menor potencial oncogénico que la del p53, dada la poca frecuencia con que se describen adenomas asociados a adenocarcinomas de vesícula (11).

Consideramos que nuestros resultados sugieren que la alteración en el p53 es un paso importante en el desarrollo de los adenocarcinomas de vesícula biliar, mientras que la lesión del p53 sería poco significativa en el desarrollo de los adenomas. Por el contrario, la sobreexpresión de ciclina D1 sería un mecanismo molecular común a ambas.

\section{BIBLIOGRAFÍA}

1. Mills S, Carter D. Sternberg diagnostic surgical pathology. $4^{\text {th }}$ ed. Lippincott; 2004.

2. Hui AM, Li X, Shi YZ, Takayama T, Torzilli G, Makuuchi M. Cyclin $\mathrm{D} 1$ overexpression is a critical event in gallbladder carcinogenesis and independently predicts decreased survival for patients with gallbladder carcinoma. Clin Cancer 2000; 6: 4272-7.

3. Ma HB, Hu HT, Di Z L, et al. Association of cyclin D1, p16 and retinoblastoma protein expression with prognosis and metastasis of gallbladder carcinoma. World J Gastroenterol 2005; 11: 744-7.

4. Roa I, Melo A, Roa J, Araya J, Villaseca M, de Aretxabala X. P53 gene mutation in gallbladder cancer. Rev Méd Chile 2000; 128: 2518 .

5. Roa I, Melo A, Roa J, et al. Gen supresor de tumores p53 en neoplasias digestivas. Rev Méd Chile 2000; 128: 1269-78.

6. Billo P, Marchegiani C, Capella C, Sessa F. Expression of p53 in gallbladder carcinoma and in dysplastic and metaplastic lesions of the surrounding mucosa. Pathologica 2000; 92: 249-56

7. Watanabe H, Date K, Itoi T, Matsubayashi H, Yokoyama N, Yamano $\mathrm{M}$, et al. Histological and genetic changes in malignant transformation of gallbladder adenoma. Ann Oncol 1999; 10: 136-9.

8. Wang SN, Chung SC, Tsai KB, Chai CY. Aberrant p53 expression and the development of gallbladder carcinoma and adenoma. Kaohsiung. J Med Sci 2006; 22: 53-9.

9. Chaube A, Tewari M, Garbyal RS, Singh U, Shukla HS. Preliminary study of p53 and c-erbB-2 expression in gallbladder cancer in Indian patients manuscript. BMC Cancer 2006; 6: 126.

10. Wistuba Miquel JF, Gazdar AF, Albores-Saavedra J. Gallbladder adenomas have molecular abnormalities different from those present in gallbladder carcinomas. Hum Pathol 1999; 30: 21-5.

11. Roa I, de Aretxabala X, Araya JC, et al. Preneoplastic lesions in gallbladder cancer. J Surg Oncol 2006; 93: 615-23. 